\title{
Reações adversas a medicamentos em pacientes com COVID-19
}

\author{
Adverse drug reactions in patients \\ with COVID-19
}

\section{Reacciones adversas a medicamentos en pacientes con COVID-19}

Renato Ferreira-da-Silva 1,2,3

doi: $10.1590 / 0102-311 \times 00228121$

Caras Editoras,

No fascículo de janeiro de 2021 de CSP foi publicado o artigo Reações Adversas a Medicamentos em Pacientes com COVID-19 no Brasil: Análise das Notificações Espontâneas do Sistema de Farmacovigilância Brasileiro 1. Melo et al. 1 fizeram um estudo transversal com etapas descritivas-explanatórias e analíticas de reações adversas a medicamentos (RAM) nos doentes COVID-19, tendo como fonte de dados os casos reportados ao Sistema Brasileiro de Farmacovigilância, da Agência Nacional de Vigilância Sanitária (ANVISA).

Os autores identificaram 631 RAM entre 402 pacientes com diagnóstico de COVID-19, sobretudo aos fármacos hidroxicloroquina (59,9\%), azitromicina $(9,8 \%)$ e cloroquina (5,2\%). A análise por sistema órgão classe (system organ class - SOC - https://www.meddra.org/About\%20MedDRA\%20/\%20 Evolution\%20/\%2027th-system-organ-class) identificou RAM graves nos sistemas cardíaco (38,8\%), gastrointestinal (14,4\%), cutâneo e subcutâneo (12,2\%) e hepatobiliar (8,9\%), em que 87,2\% das RAM foram classificadas como prováveis ou possíveis.

Os resultados publicados pelos autores são concordantes com outro estudo apresentado pela Unidade de Farmacovigilância do Porto (UFPorto) - Sistema Português de Farmacovigilância, no ISPOR 2021 - The Professional Society for Health Economics and Outcomes Research Annual Meeting e publicado no Value in Health ${ }^{2}$. A nossa análise identificou, para o período de março de 2020 a dezembro de 2020, 147 RAM, das quais 61 (41,5\%) à hidroxicloroquina e 87 (58,5\%) ao remdesivir. As cinco RAM mais reportadas com a hidroxicloroquina foram a colestase $(23,2 \%)$, lesão hepatobiliar $(14,7 \%)$, condição agravada (8,4\%), pancitopenia (4,2\%) e prolongamento do intervalo QT (4,2\%). Por outro lado, as cinco RAM mais reportadas com o remdesivir foram o aumento das transaminases $(21,7 \%)$, insuficiência renal (9,6\%), aumento da alanina aminotransferase (7\%), aumento da creatinina no sangue (4,35\%) e bradicardia (4,35\%). À semelhança do trabalho publicado por Melo et al., a média de idades foi também superior a 60 anos, sendo mais acentuada nos doentes com RAM à hidroxicloroquina (68 anos; $\sigma=16$ anos) em comparação com os doentes com RAM ao remdesivir (62 anos; $\sigma=18$ anos).

O perfil de RAM parece ser concordante em ambos os estudos, sobretudo no sistema hepatobiliar e cardíaco, associado a uma média de idades superior a 60 anos. Embora o estudo da UFPorto não tenha feito a análise por tipo de notificador, sabemos que o histórico de notificação é mais significati-
1 Unidade de

Farmacovigilância do Porto, Infarmed, I.P.) Universidade do Porto, Porto, Portugal. 2 Faculdade de Medicina, Universidade do Porto, Porto, Portugal. 3 CINTESIS - Centro de Investigação em Tecnologia e Serviços de Saúde, Porto, Portugal.

Correspondência R. Ferreira-da-Silva Unidade de Farmacovigilância do Porto, Infarmed, I.P./Faculdade de Medicina, Universidade do Porto.

Rua Doutor Plácido da Costa, 4200-450 Porto, Portugal. renato.ivos@gmail.com 
vo entre a classe médica portuguesa, ao contrário do reportado pelo estudo do Brasil, com 81,9\% dos casos de RAM a serem notificados por farmacêuticos.

$\mathrm{O}$ estudo de Melo et al. traduz-se em uma enorme contribuição na geração de real world evidence na fase de pós-comercialização dos medicamentos, muitos deles a serem utilizados fora das indicações terapêuticas aprovadas pelas agências reguladoras (utilização off-label). Os sistemas de farmacovigilância têm a responsabilidade de receber, validar, analisar, solicitar dados adicionais, codificar e colaborar na imputação de causalidade de todos as suspeitas de RAM, independentemente da indicação terapêutica desses medicamentos, doença ou condição clínica. Os sistemas de farmacovigilância, por intermédio das suas unidades regionais, têm a responsabilidade de instituir abordagens de Farmacovigilância passiva e ativa, identificando e notificando eventuais suspeitas de RAM. Adicionalmente, os sistemas de farmacovigilância devem ser capazes de identificar casos de suspeitas de RAM raras, graves e inesperadas, analisando e discutindo em equipes multidisciplinares, dando um contributo na publicação médica, seguindo os princípios éticos e de proteção de dados clínicos pessoais.

A Farmacovigilância é reconhecida como uma área prioritária na saúde pública a nível global pela Organização Mundial da Saúde (OMS) desde 1963. Atualmente, no período pandêmico motivado pela COVID-19, a Farmacovigilância constitui uma área de enorme importância, contribuindo com dados de segurança pós-comercialização, particularmente importantes à promoção da efetividade e segurança dos medicamentos. Com isto, espera-se melhores decisões clínicas e regulatórias.

\section{Informação adicional}

ORCID: Renato Ferreira-da-Silva (0000-00016517-6021).

\section{Agradecimentos}

Esta carta foi financiada por fundos nacionais e comunitários do Fundo Social Europeu (FSE), através da Fundação para a Ciência e a Tecnologia (Portugal), no âmbito da bolsa de doutoramento 2020.10231.BD (DOCTORATES 4 COVID-19) de Renato Ferreira-da-Silva.
1. Melo JRR, Duarte EC, Moraes MV, Fleck K, Silva ASN, Arrais PSD. Reações adversas a medicamentos em pacientes com COVID-19 no Brasil: análise das notificações espontâneas do sistema de farmacovigilância brasileiro. Cad Saúde Pública 2021; 37:e00245820.

2. Ivos R, Ribeiro-Vaz I, Polónia J, Silva A. PDG17 Adverse drug reactions associated to hydroxychloroquine and remdesivir in COVID-19 patients: analysis of reported ADR to the Portuguese Pharmacovigilance System. Value Health 2021; 24 Suppl 1:S90. 